\title{
Curcumin prevents strokes in stroke-prone spontaneously hypertensive rats by improving vascular endothelial function
}

\author{
Cong Lan ${ }^{1,2+}$, Xinjian Chen ${ }^{1,2,3 \dagger}$, Yuxun Zhang ${ }^{1,2}$, Wei Wang ${ }^{1,2}$, Wei Eric Wang ${ }^{1,2}$, Yukai Liu ${ }^{1,2}$, Yue Cai ${ }^{1,2,4}$,
} Hongmei Ren ${ }^{1,2}$, Shuo Zheng ${ }^{1,2}$, Lin Zhou ${ }^{1,2^{*}}$ and Chunyu Zeng ${ }^{1,2^{*}}$ (D)

\begin{abstract}
Background: Antioxidants have shown great promise in stroke prevention. Diarylheptanoids (also known as diphenylheptanoids) are a small class of plant secondary metabolites that possess antioxidant activity greater than that of a-tocopherol. Curcumin is the best known member and is mainly extracted from turmeric. This study aimed to explore whether curcumin has a preventive effect on stroke.

Methods: Stroke-prone spontaneously hypertensive rats (SHRsp) were randomly divided into control group $(n=10)$ and curcumin group $(\mathrm{n}=10)$, and saline or curcumin $(100 \mathrm{mg} / \mathrm{kg} /$ day) was administrated daily. Vascular endothelial function was examined by the relaxation of the artery in response to acetylcholine (ACH). The levels of reactive oxygen species (ROS) and nitric oxide (NO) were measured by using dihydroethidium (DHE) and 4, 5diaminofluorescein (DAF-2 DA), respectively. The expression of uncoupling protein 2 (UCP2) was examined by RT-PCR and immunoblotting.

Results: Administration of curcumin significantly delayed the onset of stroke and increased the survival of SHRsp, which was ascribed to decreased ROS and improved endothelial dependent relaxation of carotid arteries. In the presence of UCP2 inhibitor genipin, both curcumin-mediated decrease of ROS and increase of NO production were blocked.

Conclusion: Our study suggests that curcumin exerts a stroke preventive effect by attenuating oxidative stress to improve vascular endothelial function, which might be associated with UCP2 signaling.
\end{abstract}

Keywords: Endothelial function, Oxidative stress, SHRsp, Stroke, UCP2

\section{Background}

Stroke is an important cause of mortality and morbidity, accompanied with heavy economic and social burden worldwide. Although great advances have been made on the therapy of stroke, mortality and disability remain high. In addition, the absolute number of people affected by stroke worldwide increases significantly over years [1]. Therefore, prevention of stroke has been a major public health priority. Preventive strategies targeting high-risk individuals including diet and lifestyle changes have been proposed.

\footnotetext{
*Correspondence: linzhou65@hotmail.com; chunyuzeng01@163.com †Equal contributors

${ }^{1}$ Department of Cardiology, Daping Hospital, Third Military Medical University, 10 Changjiangzhilu Road, Yuzhong District, Chongqing 400042, China

Full list of author information is available at the end of the article
}

Recently, mounting evidence showed that increase in the consumption of fruits is associated with reduced risk of stroke [2, 3]. However, the potential mechanism of dietary factors on stroke is not fully understood.

Among many dietary components, curcumin is a safe, natural polyphenol compound isolated from the plant Curcuma longa, which is a widely cultivated plant in the tropical regions of Asia. Curcumin is well recognized as a dietary spice for a long time and exerts a wide spectrum of biological functions including anti-cancer [4], cardioprotection [5], anti-inflammatory [6], antioxidant [7-9] and neuroprotection [10-12]. Notably, beneficial effects of curcumin on stroke have been suggested. Curcumin could decrease risk factors of stroke, such as lowering total cholesterol, boosting high density 
lipoprotein cholesterol level [13] and inhibiting platelet aggregation [14]. Thus, these properties make curcumin a potential drug for stroke prevention. Previous studies show that curcumin could attenuate ischemic strokeinduced brain injury due to its antioxidant activity [15]. It's protective effect against stroke was also reported to be associated with its epigenetic modulatory properties [16], and neurogenesis by activating the Notch signaling pathway [17]. However, most of these studies focused on therapy, not prevention of stroke, whether or not curcumin prevents stroke occurrence is not know. Therefore, we hypothesize that curcumin plays a preventive effect on stroke. In the present study, SHRsp were used as the disease model due to its marked blood pressure elevation and predisposition in developing stroke. We showed the preventive effect of curcumin on stroke in SHRsp.

Vascular endothelial dysfunction plays critical role in pathogenesis of stroke. Vascular endothelial dysfunction, as reflected by impaired endothelium-dependent dilation (EDD), is associated with vascular oxidative stress [18]. Endothelium-derived NO, produced by the enzyme eNOS through oxidative conversion of L-arginine to L-citrulline, is important for EDD. It has been demonstrated that an increase in phosphorylation of eNOS delays the onset of stroke and increases the survival in SHRsp. Notably; curcumin was demonstrated to improve endothelial dysfunction and vascular remodeling by raising $\mathrm{NO}$ availability and reducing oxidative stress [19-21]. Our further study demonstrated the mechanisms underlying the protective effect of curcumin on stroke, and found that it is depend on UCP2 mediated anti-oxidant and NO production.

\section{Methods}

All experiments adhered to the ARRIVE guidelines and were approved by the Third Military Medical University Animal Use and Care Committee.

\section{Animal}

Male SHRsp, at 7 weeks of age, were obtained from the Shanghai Laboratory Animal Centre, Chinese Academy of Science. Rats were housed in the Animal Centre Care of Daping Hospital with a $12 \mathrm{~h}$ light/dark cycle and had ad libitum access to clean drinking water and rodent chow. After one week acclimation, the SHRsp were randomly divided into two groups: control group (saline) and curcumin group (100 mg/kg/day [22], gavage, Sigma-Aldrich, St. Louis, MO). Meantime, the SHRsp were fed with rodent chow containing $4 \%$ sodium chloride to induce stroke [23] until death. The blood pressure of the rats was measured by the tail-cuff method using a sphygmomanometer once every 2 weeks [24] (Model MLT 1030; Power Laboratory; AD Instruments, Sydney, Australia). For additional experiments after 4 weeks from the start of stroke-inducing, the rats were anesthetized with an intraperitoneal (IP) injection of pentobarbital sodium $(60 \mathrm{mg} / \mathrm{kg})$ and the carotid and basilar arteries were collected. The brain and blood samples were immediately harvested and stored at $-80^{\circ} \mathrm{C}$ until use.

\section{Assessment of arteries relaxation in vitro}

Arterial relaxation was measured using the carotid arteries of rats as previously described by Zhong MF et al. [25] Briefly, after the rats were anesthetized with an intraperitoneal injection of pentobarbital sodium $(60 \mathrm{mg} /$ $\mathrm{kg}$ body weight), the carotid arteries were carefully dissected and immediately placed in cold Krebs-Henseleit buffer (KHB) to remove the fat and connective tissues. They were then cut into ring segments with a length of 2-3 $\mathrm{mm}$. One end of the ring was mounted on an organ bath with $95 \% \mathrm{O}_{2}$ and $5 \% \mathrm{CO}_{2}$ at $37{ }^{\circ} \mathrm{C}$ and the other end was connected to an isometric force transducer for tension measurement. After $60 \mathrm{~min}$ of equilibration under a resting tension of $0.5 \mathrm{~g}$ for carotid artery ring, the rings were incubated twice with $\mathrm{KCl}(60 \mathrm{mmol} / \mathrm{L})$ to test for viability. The vasoconstriction or vasodilatation reaction to phenylephrine (PHE, $10^{-10}-10^{-5} \mathrm{mmol} / \mathrm{L}$, Sigma-Aldrich), acetylcholine (ACH, $10^{-9}-10^{-5} \mathrm{mmol} / \mathrm{L}$, Sigma-Aldrich) and sodium nitroprusside (SNP, $10^{-9}-10$ ${ }^{-5} \mathrm{mmol} / \mathrm{L}$ ) respectively were determined.

\section{Dyhidroethidium (DHE) staining}

To assess superoxide production, the basilar artery was carefully and quickly isolated from SHRsp, as described previously by Zhu J et al. [26] After removal of connective tissues, the basilar artery was immediately frozen in embedding medium, cut into $8.0 \mu \mathrm{m}$ thick sections and placed on chilled microscope slides. The samples were incubated in physiological saline solution (PSS) containing $10 \mu \mathrm{mol}$ dihydroethidium (DHE; Sigma-Aldrich) for $30 \mathrm{~min}$ at $37{ }^{\circ} \mathrm{C}$ in the dark room. The vessels were then washed twice with PSS and placed under a fluorescence microscope (Nikon TE2000; Nikon Corporation, Tokyo, Japan). Images were obtained by using an excitation wave length of 520 to $540 \mathrm{~nm}$ and a rhodamine emission filter and the fluorescence intensity was analyzed. The level of superoxide in human umbilical vein endothelial cells (HUVECs) was analyzed similarly as described above.

\section{Measurement of NO level}

NO levels in the vessels were assessed using DAF-2 DA (4, 5-diaminofluorescein, Sigma-Aldrich), as described by Donato et al. previously [6]. Arterial rings were incubated in $5 \mu \mathrm{mol} / \mathrm{l}$ DAF-2DA in the dark room for $30 \mathrm{~min}$ at $37^{\circ} \mathrm{C}$ in PSS. Once the dye loading was completed, the vessels were rinsed three times with PSS. To measure the DAF fluorescence, the glass slides were placed under a Nikon E 600 fluorescence microscope (Nikon TE2000; Nikon Corporation, Tokyo, Japan) outfitted with a $40 \times$ PlanFluor objective and a fluorescein 
isothiocyanate filter set. The signals were acquired by using the software NIS-Elements 3.0 (Nikon), and the fluorescence intensity was assessed.

Accumulation of nitrate and nitrite in the blood was recognized as the end products of NO metabolism. The levels of nitrate and nitrite were measured as previously described in the method of Nakmareong $S$ et al. [27] Briefly, plasma samples were deproteinized by ultrafiltration using centrifugal concentrators (Pall Corp., Ann Arbor, MI, USA). Then sample was mixed with $0.2 \mathrm{U} / \mathrm{ml}$ nitrate reductase and $1.2 \mu \mathrm{M}$ NADPH, $4 \mathrm{mM}$ glucose-6phosphate disodium, $1.28 \mathrm{U} / \mathrm{mL}$ glucose- 6 phosphate dehydrogenase. After being incubated for $30 \mathrm{~min}$ at $30{ }^{\circ} \mathrm{C}$, the mixture was exposed to Griess solution ( $4 \%$ sulfanilamide in $0.3 \%$ napthylenediamine dihydrochloride) for $15 \mathrm{~min}$. The light absorbance of the sample was measured on a spectrophotometer (Thermo Scientific) at $450 \mathrm{~nm}$.

\section{Measurement of plasma malondialdehyde and superoxide} dismutase

The concentration of plasma malondialdehyde (MDA) was calculated using the thiobarbituric acid (TBA) method, complying with the manufacturer's protocol (Beyotime Institute of Biotechnology, Beijing, China). Plasma superoxide dismutase (SOD) activity was assayed using a $\mathrm{Cu} / \mathrm{Zn}$-SOD and Mn-SOD Assay Kit with WST8 (Beyotime Institute of Biotechnology, Beijing, China) according to manufacturer's protocol.

\section{RT-PCR}

RT-PCR was conducted as previously described in the method of Huang $\mathrm{H}$ et al. [28] A total of $1.0 \mu \mathrm{g}$ of RNA extracted from carotid artery tissues was used to synthesize cDNA that served as the template for amplification of the uncoupling protein 2 (UCP2). $\beta$-actin was used as an endogenous standard. For UCP2, the forward primer was 5'ACCATTGCACGAGAGGAAGG-3' and the reverse primer was 5'- TCTTGACCACATCAACGGGG -3'. For $\beta$ actin, the forward primer was 5'-GTGGGTATGGGTCAGAAGGA-3' and the reverse primer was 5'-AGCGCG TAACCCTCATAGAT-3'. The amplification was performed under the following conditions: 35 cycles of denaturation for $2 \mathrm{~min}$ at $94{ }^{\circ} \mathrm{C}$, annealing for $30 \mathrm{~s}$ at $62{ }^{\circ} \mathrm{C}$, and extension for $45 \mathrm{~s}$ at $72{ }^{\circ} \mathrm{C}$. Comparison of gene expression was performed using the $\Delta \Delta C T$ method.

\section{Cell culture and treatment}

HUVECs were purchased from American Type Culture Collection (ATCC, Manassas, VA), cultured in
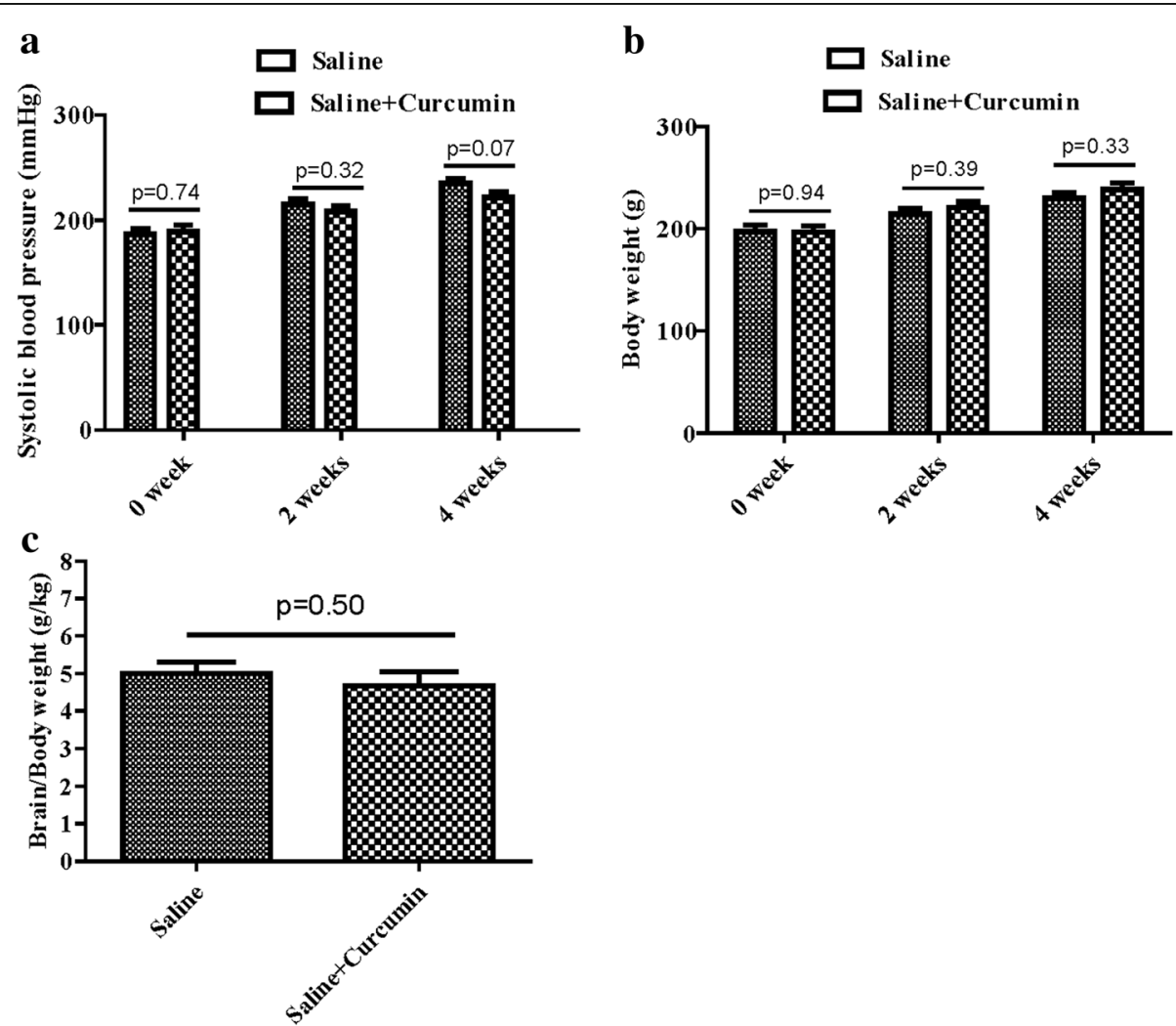

Fig. 1 Characteristics of animals. System blood pressure (a), body weight (b), the ratio of brain (g)/body weight(kg) (c) were indicated from SHRsp with or without treatment by curcumin (100 mg/kg/day, $n=10)$ 
Table 1 General characteristics of rats in saline group and saline + curcumin group

\begin{tabular}{|c|c|c|c|c|c|}
\hline & \multicolumn{2}{|c|}{ Saline $(N=10)$} & \multicolumn{2}{|c|}{ Saline+Curcumin $(N=10)$} & \multirow[t]{2}{*}{$P$ value } \\
\hline & $\overline{M e a n}$ & SEM & Mean & SEM & \\
\hline \multicolumn{6}{|c|}{ Systolic blood pressure (mmHg) } \\
\hline 0 week & 186.30 & 5.71 & 189.10 & 6.13 & 0.74 \\
\hline 2 weeks & 215.50 & 4.56 & 208.00 & 5.77 & 0.32 \\
\hline 4 weeks & 235.30 & 4.57 & 221.50 & 5.64 & 0.07 \\
\hline \multicolumn{6}{|l|}{ Body weight (g) } \\
\hline 0 week & 197.70 & 5.98 & 196.80 & 5.83 & 0.94 \\
\hline 2 weeks & 214.50 & 4.81 & 220.90 & 5.54 & 0.39 \\
\hline 4 weeks & 229.80 & 5.56 & 238.30 & 6.50 & 0.33 \\
\hline Brain/Body weight (g/kg) & 5.01 & 0.29 & 4.69 & 0.37 & 0.50 \\
\hline
\end{tabular}

Dulbecco's modified eagle medium (DMEM) with $10 \%$ fetal bovine serum (FBS) in a humid atmosphere of $5 \% \mathrm{CO}_{2}$ and $95 \%$ air at $37{ }^{\circ} \mathrm{C}$. Cells between passage 3-5 were treated with $\mathrm{H} 2 \mathrm{O} 2$ at different concentrations $(10 \mu \mathrm{mol} / \mathrm{L}, 20 \mu \mathrm{mol} / \mathrm{L}, 50 \mu \mathrm{mol} / \mathrm{L}, 100 \mu \mathrm{mol} / \mathrm{L})$ for $24 \mathrm{~h}$ with or without pretreatment with curcumin $(0.01,0.05,0.1,0.5,1$ and $5 \mu \mathrm{mol} / \mathrm{L})$ for $2 \mathrm{~h}$. Then cells were used for additional experiments.

\section{Cell viability}

The Cell Counting Kit-8 (CCK-8) assay was performed to assess cell viability as previously described in the method of Han J et al. [29] In brief, cells were cultured in 96-well cell culture plate and pretreated with curcumin for $2 \mathrm{~h}$ and then treated with various concentrations of $\mathrm{H} 2 \mathrm{O} 2$ for $24 \mathrm{~h}$. Then after the medium was removed, $100 \mu \mathrm{l}$ of fresh mixture (free serum DMEM $90 \mu \mathrm{l}$ and $10 \mu \mathrm{l}$ CCK-8 assay solution) was added to each well and incubated for an additional hour. The optical densities of the cells were assessed using a spectrophotometer (Thermo Scientific) at $450 \mathrm{~nm}$. The cell viability was expressed as a ratio of optical density of treated versus control wells.

\section{Immunoblotting}

Immunoblotting was performed as previously reported by Yang J et al. [30] Briefly, after boiling the homogenates in sample buffer $(35 \mathrm{mmol} / \mathrm{L}$ Tris- $\mathrm{HCl}, \mathrm{pH} 6.8,4 \%$ SDS, 9.3\% dithiothreitol, $0.01 \%$ bromophenol blue, 30\% glycerol) at $95{ }^{\circ} \mathrm{C}$ for $5 \mathrm{~min}, 50 \mu \mathrm{g}$ of protein were separated by SDS-PAGE (10\% polyacrylamide), and then electroblotted onto nitrocellulose membranes (Bio-Rad). The blots were blocked overnight with $5 \%$ nonfat dry milk in PBST $(0.05 \%$ Tween 20 in $10 \mathrm{mmol} / \mathrm{l}$ phosphate buffered (isotonic) saline) at $4{ }^{\circ} \mathrm{C}$ under constant shaking, then incubated with goat anti-UCP2 (Sigma-Aldrich, 1:250), rabbit anti-GAPDH (Sigma-Aldrich, 1:500) overnight in the cold-room at $4{ }^{\circ} \mathrm{C}$. The membranes were then further incubated with infrared- labeled secondary antibodies (rabbit anti-goat or donkey anti-rabbit IRDye 800, Li-Cor Biosciences, Lincoln, NE) added to bind to the primary antibody at room temperature for $1 \mathrm{~h}$. The
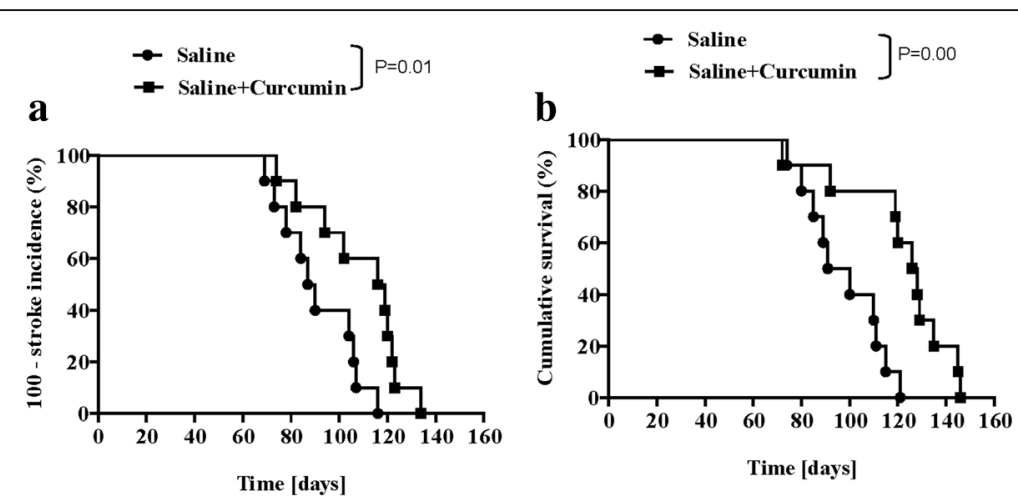

Fig. 2 Effects of curcumin on incidence of stroke (a) and survival time (b) from SHRsp. The product limit (Kaplan-Meier) estimate of the cumulative stroke and survival was assessed with the log-rank test to evaluate for significant differences in stroke and survival. $(\mathrm{n}=10)$ 

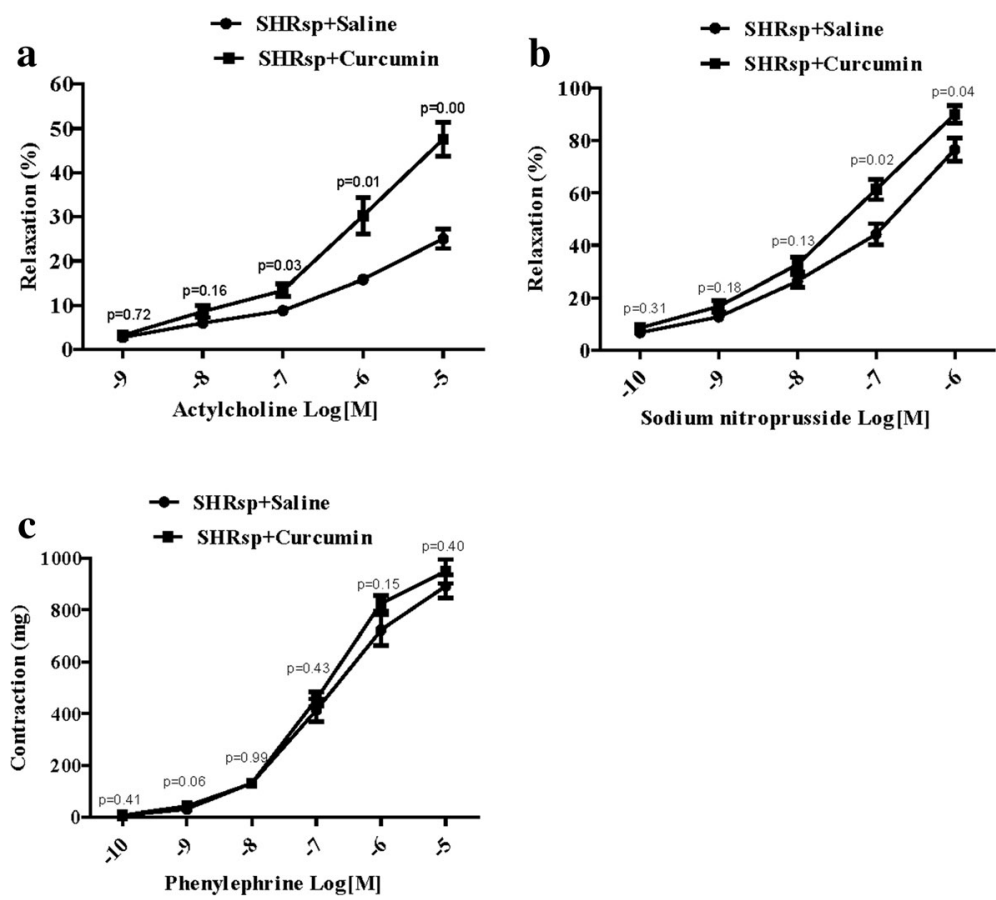

Fig. 3 Effects of curcumin on carotid artery function in SHRsp. SHRsp was fed with curcumin (100 mg/kg/day,4 weeks); carotid artery from SHRsp was exposed to acetylcholine ( $\mathrm{ACH}, n=5$ each group, a), sodium nitroprusside (SNP, $n=5$ each group, b) and phenylephrine (PHE, $n=6$ each group, c), the vasodilation or vasoconstriction was determined

membranes were washed three times with PBST. The bound complex was detected using the Odyssey Infrared Imaging System (Li-Cor Biosciences). The images were analyzed using the Odyssey Application Software to obtain the integrated intensities.

\section{Statistical analysis}

The data are expressed as mean \pm SEM. Comparison within groups were made by repeated measures analysis of variance with Duncan's ad hoc test; comparison among groups(or $t$ test when only two groups were compared) were made by factorial analysis of variance using Duncan's ad hoc test. Stroke and survival curves were made using the Kaplan-Meier method and compared using the log-rank test. Significance was defined as a value of $P<0.05$.

\section{Results}

\section{General characteristics}

To investigate the effect of curcumin on general characteristics of SHRsp, we measured blood pressure and body weight at 0,2 and 4 weeks after curcumin treatment. Results showed that curcumin treatment did not influence blood pressure ( $n=10$ each group; Fig. 1a and Table 1), body weight ( $n=10$ each group; Fig. $1 \mathrm{~b}$ and Table 1) and the ratio of brain weight (g)/body weight $(\mathrm{kg})$ of SHRsp ( $\mathrm{n}=10$ each group; Fig. $1 \mathrm{c}$ and Table 1).
Table 2 Comparison of arteries relaxation in vitro between saline group and saline + curcumin group

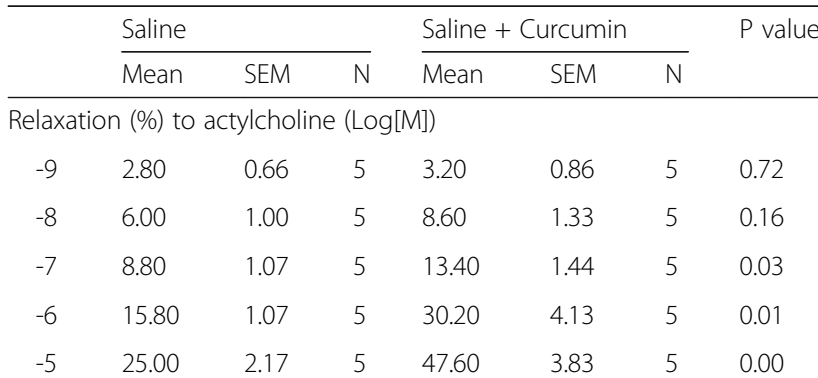

Relaxation (\%) to sodium nitroprusside (Log[M])

$\begin{array}{llllllll}-10 & 6.80 & 1.39 & 5 & 8.60 & 0.93 & 5 & 0.31 \\ -9 & 12.80 & 1.56 & 5 & 16.80 & 2.22 & 5 & 0.18 \\ -8 & 26.40 & 2.38 & 5 & 32.60 & 2.84 & 5 & 0.13 \\ -7 & 44.20 & 4.09 & 5 & 61.40 & 3.93 & 5 & 0.02 \\ -6 & 76.60 & 4.37 & 5 & 90.00 & 3.36 & 5 & 0.04\end{array}$

Contraction $(\mathrm{mg})$ to phenylephrine (Log[M])

\begin{tabular}{llllllll}
-10 & 6.83 & 0.79 & 6 & 8.33 & 1.54 & 6 & 0.41 \\
-9 & 30.83 & 2.71 & 6 & 42.17 & 4.54 & 6 & 0.06 \\
-8 & 130.17 & 16.80 & 6 & 130.00 & 4.06 & 6 & 0.99 \\
-7 & 413.50 & 43.84 & 6 & 456.17 & 28.35 & 6 & 0.43 \\
-6 & 722.67 & 58.53 & 6 & 825.67 & 31.08 & 6 & 0.15 \\
-5 & 891.67 & 45.46 & 6 & 948.83 & 46.27 & 6 & 0.40 \\
\hline
\end{tabular}




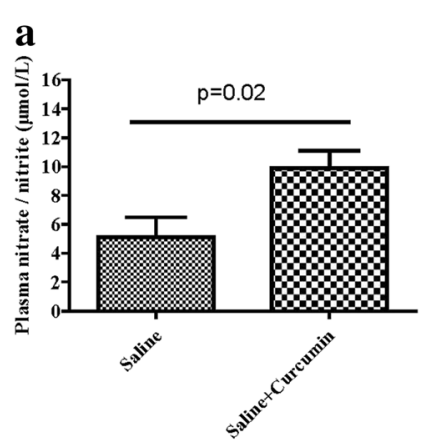

b
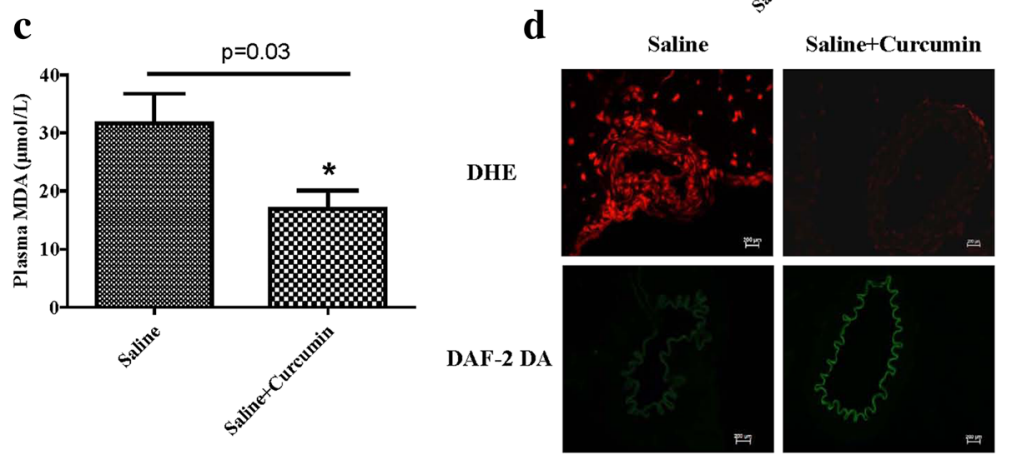

Fig. 4 Effects of curcumin on NO and ROS accumulation in the basilar artery and plasma from SHRsp. SHRsp was fed with or without curcumin $(100 \mathrm{mg} / \mathrm{kg} /$ day, 4 weeks), the plasma levels of nitrate/nitrite (a), SOD (b) and MDA (c) were determined by assay kits $(n=8$ each group). After the basilar arteries were isolated from SHRsp, NO and ROS expressions were determined by DAF-2 AF fluorescence (green, $n=3$ ) and DHE staining $($ red, $n=3)(\mathbf{d})$

\section{Curcumin treatment delayed the onset of stroke and increased survival time}

To investigate whether curcumin treatment has a preventive effect on stroke, we observed the occurrence of stroke and death in all SHRsp with or without curcumin treatment. Compared to the saline group, curcumin significantly delayed the onset of stroke $(\mathrm{n}=10$ each group; Figs. 2a), which indicates preventive effect on stroke. Meanwhile, curcumin treatment led to a remarkable increase in survival time (121.20 \pm 7.29 days versus 97.60 \pm 5.09 days, $\mathrm{n}=10$ each group, $P<0.05$; Figs. $2 \mathrm{~b}$ ), which may indicate protective effect on stroke.

\section{Curcumin treatment ameliorated arterial dysfunction in SHRsp}

Due to the role of the artery in the stroke, we checked arterial function in the carotid arteries of SHRsp. The results showed that the relaxation of carotid arteries response to $\mathrm{ACH}$ and SNP was in a dose-dependent manner. Compared with saline SHRsp, administration of curcumin significantly enhanced the relaxation of carotid artery response to $\mathrm{ACH}$ and SNP ( $n=5$ each group; Fig. 3a, b and Table 2), but did not change its response to PHE ( $n=6$ each group; Fig. $3 c$ and Table 2).

\section{Curcumin treatment increased the NO levels, but decreased ROS production in basilar arterial wall and plasma from SHRsp}

Due to the role of ROS in the pathogenesis of stroke, we checked the ROS in the plasma and artery. The results showed that curcumin increased plasma nitrate/nitrite levels and plasma SOD activity ( $n=8$ each group; Fig. 4a, $\mathrm{b}$ and Table 3) and decreased plasma MDA levels $(\mathrm{n}=8$ each group; Fig. 4c and Table 3). The anti-oxidant effects

Table 3 Comparison of plasma nitrate/nitrite, SOD and MDA between saline group and saline + curcumin group

\begin{tabular}{|c|c|c|c|c|c|c|c|}
\hline & \multicolumn{3}{|l|}{ Saline } & \multicolumn{3}{|c|}{ Saline + Curcumin } & \multirow[t]{2}{*}{$P$ value } \\
\hline & Mean & SEM & N & Mean & SEM & $\mathrm{N}$ & \\
\hline Plasma nitrate / nitrite $(\mu \mathrm{mol} / \mathrm{L})$ & 5.11 & 1.38 & 8 & 9.91 & 1.20 & 8 & 0.02 \\
\hline Plasma SOD (U/L) & 3.05 & 0.56 & 8 & 4.94 & 0.56 & 8 & 0.03 \\
\hline Plasma MDA ( $\mu \mathrm{mol} / \mathrm{L})$ & 31.69 & 5.06 & 8 & 17.01 & 3.05 & 8 & 0.03 \\
\hline
\end{tabular}



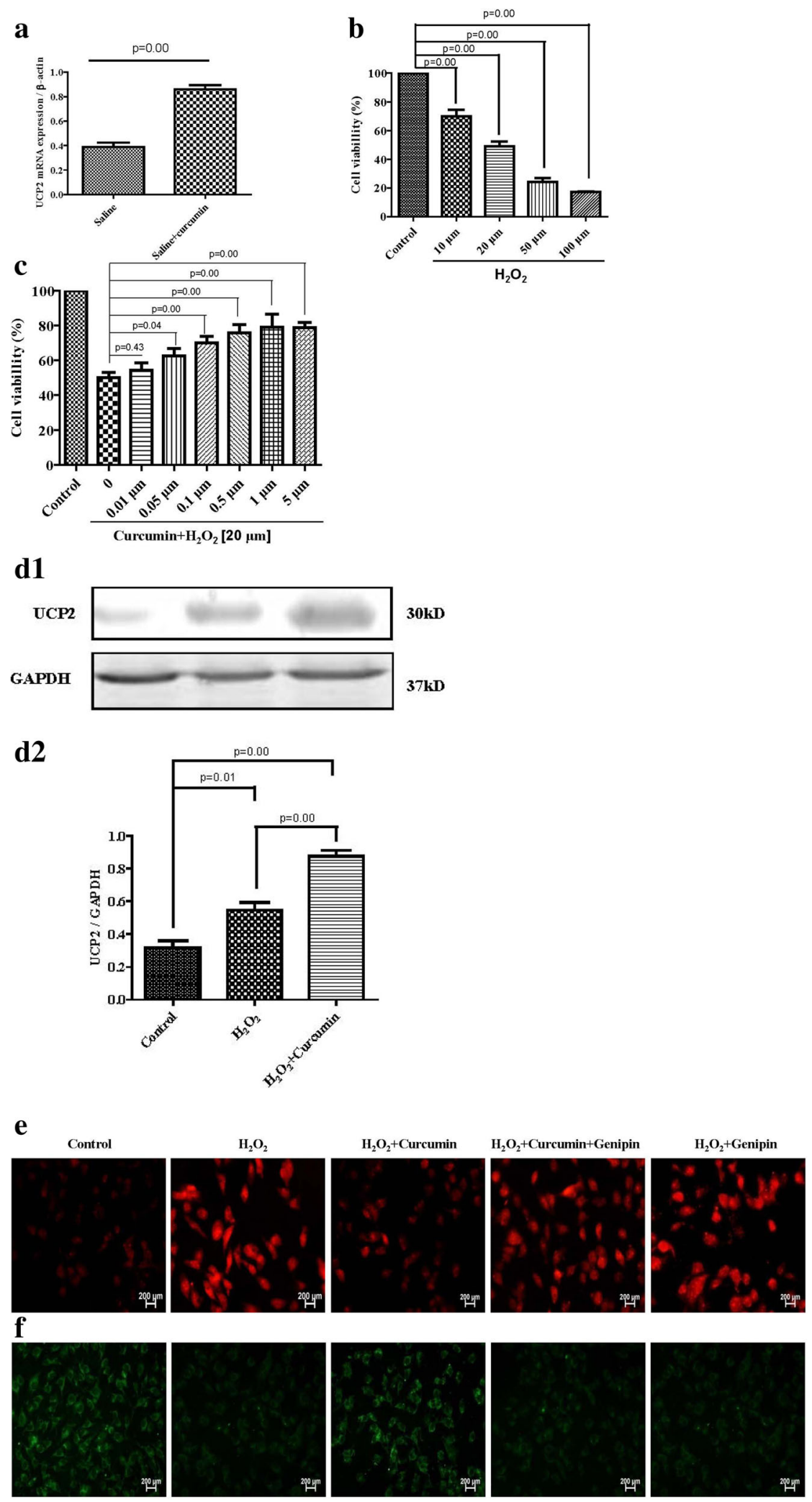

Fig. 5 (See legend on next page.) 
(See figure on previous page.)

Fig. 5 Role of UCP2 on the regulation of curcumin on ROS and NO accumulation in HUVECS a: SHRsp was fed with curcumin (100 mg/kg/day) for 4 weeks, expression of UCP2 mRNA in carotid artery SHRsp was determined by RT-PCR, Result was indicated as ratio of UCP2 and $\beta$-actin. $(n=6)$. b: HUVECs were exposed to different doses of $\mathrm{H}_{2} \mathrm{O}_{2}(10,20,50,100 \mu \mathrm{mol})$ for $24 \mathrm{~h}$. Cell viability was measured by CCK-8 assay $(n=7)$. c: HUVECs were pre-treated with curcumin $(0.01,0.05,0.1,0.5,1,5 \mu \mathrm{mol} / \mathrm{L})$ for $2 \mathrm{~h}$ and then co-incubated with $\mathrm{H}_{2} \mathrm{O}_{2}(20 \mu \mathrm{mol} / \mathrm{L})$ for $24 \mathrm{~h}$. The cell viability was measured by CCK-8 assay $(n=7)$. d-f: HUVECs were exposed to different reagents $\left(\mathrm{H}_{2} \mathrm{O}_{2} 20 \mu \mathrm{mol} / \mathrm{L}\right.$, curcumin $1 \mu \mathrm{mol} / \mathrm{L}$, genipin $10 \mu \mathrm{mol} / \mathrm{L})$. UCP2 expression was examined by western blot (d). Representative images of ROS (e) and NO (f) staining were shown (D: $\mathrm{n}=5 ; \mathrm{E}$ and $\mathrm{F}$ were repeated at least three times)

were also investigated in the artery. We measured NO and ROS in the artery instead of SOD and MDA in the plasma, and found that curcumin increased NO levels, but decreased ROS levels in the basilar artery wall $(n=3$ each group; Fig. 4d).

\section{Role of UCP2 on the curcumin protection in artery}

UCP2 is a member of the mitochondrial anion carrier family and a physiological regulator of mitochondrial ROS generation [31]. To check whether UCP2 is involved in the anti-oxidative effect of curcumin, UCP2 expression was determined by RT-PCR. Results showed that curcumin significantly increased UCP2 mRNA levels in the carotid artery from SHRsp $(n=6$ each group; Fig. 5a).

The effect of curcumin on UCP2 was also confirmed in the HUVECs using $\mathrm{H}_{2} \mathrm{O}_{2}$ to simulate oxidative stress in vitro. Results showed that $\mathrm{H}_{2} \mathrm{O}_{2}$ significantly decreased cell viability, which was attenuated by curcumin in a concentration-dependent manner ( $n=7$ each group; Fig. $5 \mathrm{~b}$ and c). Moreover, the UCP2 expression was promoted by curcumin $(n=5$ each group; Fig. 5d). Consistent with the in-vivo study, curcumin increased NO levels, decreased ROS levels in the HUVECs $(n=3$ each group; Fig. 5e and f). However, in the presence of UCP2 inhibitor, genipin $(10 \mu \mathrm{mol} / \mathrm{L})$, the effects of curcumin on ROS and $\mathrm{NO}$ were blocked (Fig. 5e and $\mathrm{f}$ ), indicating that UCP2 is an important signaling factor for the antioxidant role of curcumin.

\section{Discussion}

In the current study, we demonstrated that curcumin exerts a preventive effect on stroke supported by a latency phenotype of SHRsp. This effect might be associated with improvement of vascular endothelial function via UCP2 mediated anti-oxidant effect.

Previous studies showed that consumption of fruits and vegetables, including apple, pear, and leafy vegetables, lowers the risk of stroke [2, 32, 33]. For example, Sparassis crispa, an edibile mushroom, has been shown to have a preventive effect for stroke through amelioration cerebrovascular endothelial dysfunction [34]. Our present study demonstrated that treatment with curcumin significantly delayed the onset of stroke and markedly increased the survival time of SHRsp, supporting that diet change may be an effective way to prevent stroke and suggesting turmeric as a good choice for patients at high risk of stroke. In this study, we did not observed the effect on blood pressure, indicating the protective effect is independent of lowering blood pressure in SHRsp.

Ameliorating the endothelial dysfunction has showed a preventive effect in SHRsp [24, 34]. It is well known that endothelial $\mathrm{NO}$ is an endogenous mediator of vasodilatation. In addition, oxidative stress also plays an important role in stroke. The decreased NO production or availability, at least in part, is due to excess superoxide anion in SHRsp [35]. Therefore, enhancement of NO level and reduction of oxidative stress might be feasible strategies to prevent stroke occurrence. Curcumin has shown its beneficial effect in improvement of endothelial function through raising nitric oxide availability and reducing oxidative stress. Our present study found that curcumin increased NO levels, decreased ROS levels in plasma and basilar artery and significantly improved endothelial dysfunction in SHRsp. This protective effect against endothelial dysfunction was consistent with previous studies [19, 21]. Notably, SNP leads to release of NO thus dilating artery and in our study EDD with SNP was promoted by curcumin. Therefore, we speculate that SNP and curcumin have synergistic effect in increasing NO levels thus combined administration might be beneficial for preventing stroke.

UCP2 is a member of the mitochondrial uncoupling proteins, belonging to the mitochondrial inner anion carrier family, which mediates the proton leak and control of superoxide [31]. Evidence suggests that UCP2 is a negative regulator of ROS generation within the mitochondrion [36]. It has been demonstrated that UCP2 inhibition leads to a significant increase of oxidative stress in endothelial cells, whereas overexpression of UCP2 inhibits endothelial cells apoptosis and ameliorates vascular endothelial function by inhibiting ROS production [37-39]. In addition, upregulation of UCP2 expression plays an important role in the restoration of hyperglycemiainduced endothelial dysfunction, which is due to the 
suppression of superoxide production and the elevation of NO production in the blood vessels and endothelial cells [40]. Therefore, we examined whether UCP2 involved in the endothelial protection of curcumin. In the presence of UCP2 blocker, the effects of curcumin on ROS and NO productions were blocked. Thus we speculate that UCP2 signaling is involved in the preventive effect of curcumin on stroke in hypertension via the decreasing ROS and increasing NO levels.

Mitochondria is suggested by many studies as a target in the therapeutic properties of curcumin in many disease [41]. In cancer state, curcumin was observed to induce permeability transition pore opening and production of ROS, thus inducing apoptosis of cancer cells and exerting anti-cancer effect [42]. On the contrary, in physiological conditions, it was observed to induce cytoprotective enzymes and ROS diminishing, thus exerting anti-oxidant effect [43]. The mechanism by which curcumin exerts its dual role in normal and cancer cells is still unknown and remains to be explored. Our study showed curcumin decreases ROS in artery and HUVECs, and this is helpful to understand the protective effect of curcumin on stroke. It should be noticed that the detailed mechanisms of curcumin on ROS production need to be investigated in the future.

\section{Conclusion}

Despite increased knowledge in controlling risk factors of stroke, how to control stroke remains challenging. Curcumin has been shown to have vascular protective benefits in the arteries. In our present study, we report that administration of curcumin significantly delayed the onset of stroke and increased the survival time for SHRsp. Interestingly, this effect is independent of a reduction in blood pressure. Instead, reduction of oxidative stress, via a UCP2/NO pathway, is involved in the preventive process.

\section{Abbreviations}

ACH: Acetylcholine; CCK-8: Cell Counting Kit-8; DAF-2 DA: 4, 5diaminofluorescein; DHE: Dihydroethidium; DMEM: Dulbecco's Modified Eagle Medium; EDD: Endothelium-dependent Dilation; FBS: Fetal Bovine Serum; HUVECs: Human Umbilical Vein Endothelial Cells; IP: Intraperitoneal; KHB: Krebs-Henseleit Buffer; MDA: Malondialdehyde; NO: Nitric Oxide; PHE: Phenylephrine; PSS: Physiological Saline Solution; ROS: Reactive Oxygen Species; SHRsp: Stroke-prone Spontaneously Hypertensive Rats; SNP: Sodium Nitroprusside; SOD: Superoxide Dismutase; TBA: Thiobarbituric Acid; UCP2: Uncoupling Protein 2

\section{Acknowledgments}

Not applicable.

\section{Funding}

These study was supported in part by grants from the National Natural Science Foundation of China $(31730043,31430043)$ and the National Basic Research Program of China (2012CB517801 and 2013CB531104). This research also received supports from Program of Innovative Research Team by National Natural Science Foundation (81721001). The funding bodies were not involved in data analysis or interpretation as well as writing or submitting the manuscript.

\section{Availability of data and materials}

The datasets used during the current study are available from the corresponding author on reasonable request.

\section{Authors' contributions}

$C Z$ and $L Z$ conceived the project and designed the experiments. $C L, X C, Y Z$, WW, WEW, YL, YC, HR, and SZ conducted experiments. CL wrote the manuscript. YZ improved English in manuscript. CZ and LZ revised the manuscript. All authors reviewed and approved the manuscript.

\section{Ethics approval and consent to participate}

This study was approved by the Third Military Medical University Animal Use and Care Committee.

\section{Consent for publication}

Not applicable.

\section{Competing interests}

The authors declare that they have no competing interests.

\section{Publisher's Note}

Springer Nature remains neutral with regard to jurisdictional claims in published maps and institutional affiliations.

\section{Author details}

${ }^{1}$ Department of Cardiology, Daping Hospital, Third Military Medical University, 10 Changjiangzhilu Road, Yuzhong District, Chongqing 400042, China. ${ }^{2}$ Chongqing Institute of Cardiology, Chongqing, China. ${ }^{3}$ Department of Cardiology, 458 Hospital of People's Liberation Army, Guangzhou, China. ${ }^{4}$ Department of Cardiology, the hospital of PLA N.O.95877, 14 branch road, Jiuquan, Gansu 735018, China.

Received: 15 August 2017 Accepted: 2 February 2018

Published online: 01 March 2018

\section{References}

1. Feigin VL, Norrving B, George MG, et al. Prevention of stroke: a strategic global imperative. Nat Rev Neurol. 2016;12:501-12.

2. Larsson SC, Virtamo J, Wolk A. Total and specific fruit and vegetable consumption and risk of stroke: a prospective study. Atherosclerosis. 2013; 227:147-52.

3. He FJ, Nowson CA, MacGregor GA. Fruit and vegetable consumption and stroke: meta-analysis of cohort studies. Lancet. 2006:367:320-6.

4. Hassan ZK, Daghestani MH. Curcumin effect on mmps and timps genes in a breast cancer cell line. Asian Pac J Cancer Prev. 2012;13:3259-64.

5. Wang NP, Wang ZF, Tootle $\mathrm{S}$, et al. Curcumin promotes cardiac repair and ameliorates cardiac dysfunction following myocardial infarction. $\mathrm{Br}$ J Pharmacol. 2012;167:1550-62.

6. Zhong Y, Liu T, Guo Z. Curcumin inhibits ox-LDL-induced MCP-1 expression by suppressing the p38MAPK and NF-KB pathways in rat vascular smooth muscle cells. Inflamm Res. 2012;61:61-7.

7. Yang Y, Duan W, Liang Z, et al. Curcumin attenuates endothelial cell oxidative stress injury through notch signaling inhibition. Cell Signal. 2013; 25:615-29.

8. Fleenor BS, Sindler AL, Marvi NK, et al. Curcumin ameliorates arterial dysfunction and oxidative stress with aging. Exp Gerontol. 2013;48:269-76.

9. Tiwari V, Chopra K. Attenuation of oxidative stress, neuroinflammation, and apoptosis by curcumin prevents cognitive deficits in rats postnatally exposed to ethanol. Psychopharmacology. 2012;224:519-35.

10. Yadav RS, Shukla RK, Sankhwar ML, et al. Neuroprotective effect of curcumin in arsenic-induced neurotoxicity in rats. Neurotoxicology. 2010;31:533-9.

11. Thiyagarajan M, Sharma SS. Neuroprotective effect of curcumin in middle cerebral artery occlusion induced focal cerebral ischemia in rats. Life Sci. 2004;74:969-85

12. Zhao J, Zhao $Y$, Zheng $W$, et al. Neuroprotective effect of curcumin on transient focal cerebral ischemia in rats. Brain Res. 2008;1229:224-32. 
13. Soni KB, Effect KR. Of oral curcumin administration on serum peroxides and cholesterol levels in human volunteers. Indian J Physiol Pharmacol. 1992;36:273-5.

14. Ovbiagele B. Potential role of curcumin in stroke prevention. Expert Rev Neurother. 2008:8:1175-6.

15. Ahmad N, Umar S, Ashafaq M, et al. A comparative study of pnipam nanoparticles of curcumin, demethoxycurcumin, and bisdemethoxycurcumin and their effects on oxidative stress markers in experimental stroke. Protoplasma. 2013;250:1327-38.

16. Kalani A, Kamat PK, Kalani K, et al. Epigenetic impact of curcumin on stroke prevention. Metab Brain Dis. 2015;30:427-35.

17. Liu S, Cao Y, Qu M, et al. Curcumin protects against stroke and increases levels of notch intracellular domain. Neurol Res. 2016;38:553-9.

18. van der Loo B, Labugger R, Skepper JN, et al. Enhanced peroxynitrite formation is associated with vascular aging. J Exp Med. 2000;192:1731-44.

19. Boonla O, Kukongviriyapan U, Pakdeechote $P$, et al. Curcumin improves endothelial dysfunction and vascular remodeling in $2 \mathrm{~K}-1 \mathrm{C}$ hypertensive rats by raising nitric oxide availability and reducing oxidative stress. Nitric Oxide. 2014:42:44-53.

20. Zhang DM, Li YC, Xu D, et al. Protection of curcumin against fructoseinduced hyperuricaemia and renal endothelial dysfunction involves NOmediated JAK-STAT signalling in rats. Food Chem. 2012;134:2184-93.

21. Ramaswami G, Chai H, Yao Q, et al. Curcumin blocks homocysteine-induced endothelial dysfunction in porcine coronary arteries. J Vasc Surg. 2004;40: 1216-22.

22. Yadav RS, Chandravanshi LP, Shukla RK, et al. Neuroprotective efficacy of curcumin in arsenic induced cholinergic dysfunctions in rats. Neurotoxicology. 2011;32:760-8.

23. Yamamoto E, Tamamaki N, Nakamura T, et al. Excess salt causes cerebral neuronal apoptosis and inflammation in stroke-prone hypertensive rats through angiotensin II-induced NADPH oxidase activation. Stroke. 2008;39: 3049-56.

24. Xu X, Wang P, Zhao Z, et al. Activation of transient receptor potential vanilloid 1 by dietary capsaicin delays the onset of stroke in stroke-prone spontaneously hypertensive rats. Stroke. 2011;42:3245-51.

25. Zhong MF, Shen WL, Wang J, et al. Paradoxical effects of streptozotocininduced diabetes on endothelial dysfunction in stroke-prone spontaneously hypertensive rats. J Physiol. 2011;589:5153-65.

26. Zhu J, Huang T, Lombard JH. Effect of high-salt diet on vascular relaxation and oxidative stress in mesenteric resistance arteries. J Vasc Res. 2007;44: 382-90.

27. Nakmareong $\mathrm{S}$, Kukongviriyapan U, Pakdeechote $\mathrm{P}$, et al. Antioxidant and vascular protective effects of curcumin and tetrahydrocurcumin in rats with L-NAME-induced hypertension. Naunyn Schmiedeberg's Arch Pharmacol. 2011;383:519-29.

28. Huang $H_{\text {, }}$ Ren $H_{\text {, }}$ Chen $C_{\text {, et al. }} D_{3}$ dopamine receptor regulation of $D_{5}$ receptor expression and function in renal proximal tubule cells. Hypertens Res. 2012;35:639-47.

29. Han J, Pan $X Y, X u Y$, et al. Curcumin induces autophagy to protect vascular endothelial cell survival from oxidative stress damage. Autophagy. 2012;8: 812-25.

30. Yang J, Han $Y$, Sun $H$, et al. (-)-epigallocatechin gallate suppresses proliferation of vascular smooth muscle cells induced by high glucose by inhibition of PKC and ERK1/2 signalings. J Agric Food Chem. 2011; 59:11483-90.

31. Krauss S, Zhang CY, Lowell BB. The mitochondrial uncoupling-protein homologues. Nat Rev Mol Cell Biol. 2005;6:248-61.

32. Oude Griep LM, Verschuren WM, Kromhout D, et al. Variety in fruit and vegetable consumption and 10-year incidence of chd and stroke. Public Health Nutr. 2012;15:2280-6.

33. Oude Griep LM, Verschuren WM, Kromhout D, et al. Raw and processed fruit and vegetable consumption and 10-year stroke incidence in a population-based cohort study in the netherlands. Eur J Clin Nutr. 2011; 65:791-9.

34. Yoshitomi H, Iwaoka $E$, Kubo M, et al. Beneficial effect of sparassis crispa on stroke through activation of AKT/eNOS pathway in brain of SHRsp. J Nat Med. 2011;65:135-41.

35. Kerr S, Brosnan MJ, McIntyre M, et al. Superoxide anion production is increased in a model of genetic hypertension: role of the endothelium. Hypertension. 1999;33:1353-8.
36. Mattiasson G, Sullivan PG. The emerging functions of UCP2 in health, disease, and therapeutics. Antioxid Redox Signal. 2006;8:1-38.

37. Tian $X Y$, Wong $W T, X u A$, et al. Uncoupling protein-2 protects endothelial function in diet-induced obese mice. Circ Res. 2012;110:1211-6.

38. Xie Z, Zhang J, Wu J, et al. Upregulation of mitochondrial uncoupling protein-2 by the AMP-activated protein kinase in endothelial cells attenuates oxidative stress in diabetes. Diabetes. 2008;57:3222-30.

39. Lee KU, Lee IK, Han J, et al. Effects of recombinant adenovirus-mediated uncoupling protein 2 overexpression on endothelial function and apoptosis. Circ Res. 2005;96:1200-7.

40. Sun J, Pu Y, Wang P, et al. TRPV1-mediated UCP2 upregulation ameliorates hyperglycemia-induced endothelial dysfunction. Cardiovasc Diabetol. 2013;12:69.

41. Wang J, Qi L, Mei L, et al. Curcumin inhibits the proliferation and induces apoptosis in HT-29 cell lines through a reactive oxygen species (ROS)dependent mechanism. Pak J Pharm Sci. 2017;30:1671-7.

42. Jayakumar S, Patwardhan RS, Pal D, et al. Mitochondrial targeted curcumin exhibits anticancer effects through disruption of mitochondrial redox and modulation of TrxR2 activity. Free Radic Biol Med. 2017;113:530-8.

43. Trujillo J, Chirino Yl, Molina-Jijon E, et al. Renoprotective effect of the antioxidant curcumin: recent findings. Redox Biol. 2013;1:448-56.

\section{Submit your next manuscript to BioMed Central and we will help you at every step:}

- We accept pre-submission inquiries

- Our selector tool helps you to find the most relevant journal

- We provide round the clock customer support

- Convenient online submission

- Thorough peer review

- Inclusion in PubMed and all major indexing services

- Maximum visibility for your research

Submit your manuscript at www.biomedcentral.com/submit 